\title{
CONNECTED LOCALLY COMPACT GROUPS: THE HOCHSCHILD KERNEL AND FAITHFULNESS OF LOCALLY BOUNDED FINITE-DIMENSIONAL REPRESENTATIONS
}

\author{
A. I. SHTERN
}

\begin{abstract}
We obtain a number of consequences of the theorem on the automatic continuity of locally bounded finite-dimensional representations of connected Lie groups on the derived subgroup of the group, as well as an analogue of Lie's theorem for (not necessarily continuous) finite-dimensional representations of connected soluble locally compact groups. In particular, we give a description of connected Lie groups admitting a (not necessarily continuous) faithful locally bounded finitedimensional representation; as it turns out, such groups are linear. Furthermore, we give a description of the intersection of the kernels of continuous finite-dimensional representations of a given connected locally compact group, obtain a generalization of Hochschild's theorem on the kernel of the universal representation in terms of locally bounded (not necessarily continuous) finite-dimensional linear representations, and find the intersection of the kernels of such representations for a connected reductive Lie group.
\end{abstract}

\section{INTRODUCTION}

Physical measurements, being of a local nature, give information on the deviation of the size of some or other quantity within the limits of the accuracy of the measuring apparatus. From this point of view, the category of topological spaces and maps of these spaces, under which each point has a neighbourhood with precompact image, is one of the rivals of the "more natural" category of topological spaces and continuous maps of them. We can consider the first part of the more than 80 -year history of research into the automatic continuity of finite-dimensional representations of connected Lie groups in this connection. Apparently the first work along these lines was E. Cartan's work [1], in which it was proved that any (not a priori continuous) finite-dimensional representation of a connected simple compact Lie group is automatically continuous. Somewhat later, van der Waerden [2] proved that any (not a priori continuous) finite-dimensional representation of a (not necessarily compact) Lie group is automatically continuous. This work all but replaces Cartan's result in the memory of the mathematical community, but in many sources (3]-8]), including the author's publication [9, Cartan's result is ascribed to van der Waerden. I am grateful to Dieter Remus [10], who drew my attention to the publications [11] and [12] in which the historical truth is established.

The related question of the automatic continuity of automorphisms, isomorphisms and homomorphisms, in particular those with a dense image, was considered by many authors ([13]-[23]), starting from 1928, but the results of these papers rely on the condition that

2010 Mathematics Subject Classification. Primary 22E15, 22C05.

Key words and phrases. Locally compact group, almost connected locally compact group, Freudenthal-Weil theorem, MAP group, semisimple locally compact group, locally bounded map.

This work was carried out with the financial support of the Russian Foundation for Basic Research (grant no. 08-01-000034) and the Programme of Support for Leading Scientific Schools (grant no. NSh1562.2008.1). 
the image of the corresponding map be dense or that the groups under consideration are algebraic, so these results cannot be directly applied to the study of finite-dimensional representations of connected Lie groups. In particular, although Tits' paper [19] (which mainly uses the methods and results from 20]) contained sufficient conditions for the automatic continuity of homomorphisms of Lie groups with a perfec 11 component of the identity without assuming that the homomorphism is locally bounded. He did, however, impose some additional assumptions, for example, that the adjoint representation of the Levi subalgebra of the Lie algebra of the group-image and the restriction of the adjoint representation of this Lie algebra to its radical do not have a common direct summand, which, of course, holds automatically for finite-dimensional representations, but a key point is that all the simple factors of the Levi subgroup are absolutely simple (that is, they have simple complexifications; see [19], p. 489, Corollary 2), and this condition does not hold for the group $\operatorname{SL}(n, \mathbb{C})$. This means that this condition cannot be directly applied to complex linear representations, in view of the fact that the Lie group $\mathrm{SL}(n, \mathbb{C})$ is not absolutely simple.

In the author's paper [9] we obtained a number of results on the automatic continuity of locally bounded finite-dimensional linear representations of connected Lie groups, which proved to be productive in investigating a number of questions about the structure of embeddings, of more general homomorphisms and representations of connected Lie groups and connected locally compact groups. Using these, in [24] we obtained a number of results on the structure of homomorphisms taking connected locally compact groups into compact groups. In this paper we consider applying the results in 9 to study the question of the existence of a faithful locally bounded (not necessarily continuous) finite-dimensional linear representation of a given locally compact group and to give a description of the Hochschild $\operatorname{kernel} \operatorname{urk}(G)$ of a given locally compact group $G$ (or of the intersection of the kernels of continuous finite-dimensional linear representations of a topological group $G$ ).

We recall a result due to Hochschild 25, which will be of importance in what follows. If $G$ is a semisimple Lie group, then the group $\operatorname{urk}(G)$ is constructed in the following way. Let $\mathfrak{g}$ be the Lie algebra of the Lie group $G$, let $\mathbb{C}$ be the complex number field and let $\mathfrak{g}^{\mathbb{C}}$ be the complexification of $\mathfrak{g}$, that is, the semisimple Lie algebra over $\mathbb{C}$ obtained as the tensor product (over the field $\mathbb{R}$ ) of the Lie algebra $\mathfrak{g}$, and $\mathbb{C}, \mathfrak{g}^{\mathbb{C}}=\mathfrak{g} \otimes_{\mathbb{R}} \mathbb{C}$. We denote by $S(\mathfrak{g})$ and $S\left(\mathfrak{g}^{\mathbb{C}}\right)$ the simply connected Lie groups with Lie algebras $\mathfrak{g}$ and $\mathfrak{g}^{\mathbb{C}}$, respectively. The embedding $\mathfrak{g} \rightarrow \mathfrak{g}^{\mathbb{C}}$ is the differential of the uniquely defined continuous homomorphism $\gamma$ of the group $S(\mathfrak{g})$ into $S\left(\mathfrak{g}^{\mathbb{C}}\right)$. The kernel $D=D(G)$ of the homomorphism $\gamma$ is a discrete central subgroup of the group $S(\mathfrak{g})$. Let $\varphi$ be the covering epimorphism of the group $S(\mathfrak{g})$ onto $G$.

Theorem I (see [25]). Let $G$ be a semisimple Lie group. Then $\operatorname{urk}(G)=\varphi(D)$. Thus, the kernel $\operatorname{urk}(G)$ of the universal representation of the semisimple connected Lie group $G$ is the image of the kernel of the canonical homomorphism $S(\mathfrak{g}) \rightarrow S\left(\mathfrak{g}^{\mathbb{C}}\right)$ under the universal covering epimorphism of $S(\mathfrak{g})$ onto $G$.

We now recall the main result in Hochschild [25].

Theorem II (see [25]). Let $G$ be a connected Lie group and let $S$ be a maximal semisimple analytic subgroup of $G$. Let $A$ be the closure in $G$ of $\operatorname{urk}(S)$ (see Theorem I). Then $\operatorname{urk}(G)$ coincides with a closed subgroup (normal divisor) $P$ of $G$ containing $A$ for which the quotient group $P / A$ is the (unique) maximal compact subgroup of the closure (in $G / A$ ) of the radical of the derived subgroup $(G / A)_{*}$ of $G / A$.

\footnotetext{
${ }^{1}$ That is, coinciding with its (possibly nonclosed) derived subgroup generated by the commutators $a b a^{-1} b^{-1}$, where $a$ and $b$ run through the group.
} 
We now give a description of the Hochschild kernel of connected locally compact groups. We use a result due to Iwasawa on local decomposition ([26, Theorem 11) from which it immediately follows that in any neighbourhood of the identity of $G$ there is a compact (not necessarily connected) normal divisor $N$ such that the quotient group $L=$ $G / N$ is a connected Lie group and $G$ is the image of some homomorphism $\varphi: \widetilde{L} \times N \rightarrow G$ with discrete kernel, where $\widetilde{L}$ is the universal covering of the Lie group $L$. We also recall that the radical $R$ of the locally compact group $G$ is the largest (closed) connected soluble normal divisor of $G$ (see, for example, [26] or [27, Theorem 3.7).

Using these objects we first obtain a very natural description of connected locally compact groups having sufficiently many continuous finite-dimensional representations. We recall that the expression 'sufficiently many' means that the corresponding family of representations separates elements of the group under consideration.

Theorem 1. Let $G$ be a connected locally compact group. Then the following conditions are equivalent:

(i) $G$ has sufficiently many continuous linear representations in finite-dimensional vector spaces; that is, the family of finite-dimensional linear representations of $G$ separates the points of $G$.

(ii) $\operatorname{urk}(G)=\left\{e_{G}\right\}$.

(iii) $G$ is the projective limit of connected linear Lie groups.

(iv) The largest noncompact (analytic) semisimple Lie subgroup $S_{1}$ of $G$ is a linear Lie group (this means, of course, that the corresponding group $\operatorname{urk}\left(S_{1}\right)$ is trivial) and the (unique) maximal compact subgroup $B$ of the closure (in $G)$ of the radical $\mathrm{rad}\left(G^{\prime}\right)$ of the derived subgroup $G^{\prime}$ of $G$ is also trivial.

We now describe the Hochschild kernel of a connected locally compact group $G$ with radical $R$. Let $[G, R]$ be the subgroup of $G$ generated by commutators of the form $g r g^{-1} r^{-1}$, where $g \in G$ and $r \in R$. The Hochschild kernel $\operatorname{urk}(G)$ of $G$ can then be described in the following way.

Theorem 2. Let $G$ be a connected locally compact group, $U$ a given neighbourhood of the identity in $G, N \subset U$ a compact (not necessarily connected) normal divisor such that the quotient group $L=G / N$ is a connected Lie group and $G$ is the image of some homomorphism $\psi: \widetilde{L} \times N \rightarrow G$ with discrete kernel, where $\widetilde{L}$ is the universal covering of the Lie group L. Let $S_{\widetilde{L}}$ be the Levi subgroup of $\widetilde{L}$, let $S$ be the image of $S_{\widetilde{L}}$ in $G$, and let $A$ be the closure (in $G$ ) of $\operatorname{urk}(S)$ (see Theorem I). Then $\operatorname{urk}(G)$ coincides with the closed subgroup (normal divisor) $P$ of $G$ containing $A$ for which the quotient group $P / A$ is the (unique) maximal compact subgroup of the closure (in $G / A$ ) of the radical of the derived subgroup $(G / A)_{*}$ of $G / A$.

This paper is organized in the following way. The necessary preliminary information is set out in $\S 2$. The criterion for a representation of a connected locally compact group to be continuous and linear (so that its Hochschild kernel is trivial; see Theorem 1) and the criterion for a connected Lie group to be linear, given in terms of (not necessarily continuous) finite-dimensional locally bounded representations (Theorem 12) are considered in $\S 3$. The theorem on the Hochschild kernel for connected locally compact groups (Theorem 2) is proved in $\S 4$. A certain generalization of Hochschild's theorem in terms of locally bounded finite-dimensional representations is obtained in $\S 5$ and a description of the intersection of the kernels of locally bounded finite-dimensional representations of a reductive connected Lie group is given in $\S 6$.

Some of the results of this paper were announced (with outlines of proofs) in [28 -33 . 


\section{Preliminary information}

We recall some definitions and results from [9] used in the proofs of this paper; all the proofs not given here can be found in 9 .

Definition 3. Let $G$ be a group, and $X$ a subset of $G$. The set $X$ is called infinitely divisible if for any element $x \in X$ and any positive integer $p$ there exists an element $y \in X$ such that $y^{p}=x$. The group $G$ is said to be locally infinitely divisible if the operation of raising to any positive power $p$ is open in the group identity; that is, the set of $p$ th powers of elements running through any neighbourhood of the identity in $G$ contains some neighbourhood of the identity in $G$.

Clearly, any Lie group is locally infinitely divisible. The next result shows that in any topological group, closures of infinitely divisible relatively compact subgroups are infinitely divisible.

Lemma 4. Let $G$ be a topological group, and $X$ a subset of $G$ with compact closure $\bar{X}$ in $G$. If $X$ is infinitely divisible, then $\bar{X}$ is infinitely divisible.

We now state one of the simplest generalizations of Lie's theorem.

Theorem III (see [9]). Let $G$ be a soluble group and suppose that each commutative factor in the composition series of $G$ is infinitely divisible. Let $\pi$ be a representation of $G$ in a finite-dimensional complex linear space $E$. If the representation $\pi$ is irreducible, then the space of the representation $\pi$ is one-dimensional.

We recall that any commutative connected locally compact group is infinitely divisible (see [9], Corollary 1), as is any compact connected group (see [6], Theorem 9.35) and the successive derived groups of a connected topological group are connected, as are the quotient groups by it; therefore any connected soluble locally compact group satisfies all the conditions of Theorem I. Hence we have the following result.

Corollary 5 (see [9], Corollary 5). 1) Any (not necessarily continuous) irreducible finite-dimensional representation of a connected soluble locally compact group is onedimensional.

2) Any (not necessarily continuous) finite-dimensional representation of a connected locally compact group has a basis in which the operators of the representation are described by upper-triangular matrices.

We shall prove a somewhat more general result, which is used below and is of interest in its own right.

Lemma 6. Let $G$ be a group, $N$ a normal divisor in $G, \pi$ an irreducible representation of $G$ in a finite-dimensional vector space $E$ and suppose that there exists a one-dimensional subspace $L \subset E$ that is invariant with respect to the restriction $\sigma$ of the representation $\pi$ to $N$ in $E$. If for any $p \in \mathbb{N}$ there exists a subset $X \subset G / N$ formed by the pth powers of the elements of $G / N$ and generating $G / N$, then all the operators of the restriction of the representation $\sigma$ of $N$ in $E$ are multiples of the identity operator in $E$.

Proof. By hypothesis, the representation $\sigma$ has a one-dimensional subrepresentation and therefore a common eigenvector $\xi \in E$, so that, in particular,

$$
\sigma(n) \xi=\lambda(n) \xi \text { for all } n \in N,
$$

where $\lambda(n) \in \mathbb{C}^{*}, n \in N$. Then

$$
\sigma(n) \pi(g) \xi=\pi(g) \sigma\left(g^{-1} n g\right) \xi=\lambda\left(g^{-1} n g\right) \pi(g) \xi, \quad n \in N, \quad g \in G ;
$$


therefore all vectors of the form $\pi(g) \xi, g \in G$, are common eigenvectors for the representation $\sigma$. All such vectors span some finite-dimensional subspace $F \subset E$ (the linear span of all vectors of the form $\pi(g) \xi, g \in G$ in a subspace of $E$ ) and, by construction, the subspace $F$ has a basis of eigenvectors of the representation $\sigma$. Of course, any set of linearly independent vectors of the form $\sigma(g) \xi, g \in G$, is finite. It follows from (2.1) that the nonzero subspace $F$ is invariant with respect to the representation $\pi$ of $G$. By hypothesis, the representation $\pi$ is irreducible, so that $F=E$. Thus, the set of distinct functions of the form $\lambda_{g}, g \in G$, is finite, where

$$
\lambda_{g}(n)=\lambda\left(g^{-1} n g\right), \quad g \in G, \quad n \in N,
$$

since $\lambda_{g}, g \in G$, are the complex-valued characters of the group $N$; then they are invariant with respect to inner automorphisms of the group $N$. Therefore $\lambda_{n}=\lambda, n \in N$. This means that the group $G / N$ acts transitively on the (nonempty) finite set $\left\{\lambda_{g} \mid g \in G\right\}$ by permutations of this finite set, and if the number of elements in this finite set is equal to $m$, then the image of the group $G / N$ is a subgroup of the symmetric group $S_{m}$, so that the order of this subgroup is a divisor of $m$ !. By hypothesis, for any $x \in X$ there exists an element $y \in G$ such that $y^{m !}=x$. Consequently, the permutation corresponding to the element $x$ is the $m$ !th power of the permutation corresponding to $y$ and therefore it is the identity permutation. Since, by hypothesis, $X$ generates the group $G / N$, it follows that all the permutations determining the transitive action of $G / N$ on $\left\{\lambda_{g} \mid g \in G\right\}$ are the identity, so that the set $\left\{\lambda_{g} \mid g \in G\right\}$ is a singleton: $\lambda_{g}=\lambda$ for all $g \in G$. This means that all the operators of the restriction of the representation $\sigma$ to the normal divisor $N$ are scalar multiples of the identity operator on $E$ (operators of multiplication by a number, $\left.\sigma(n)=\pi(n)=\lambda(n) 1_{E}, n \in N\right)$.

The following result describes the structure of any (not necessarily continuous) locally bounded irreducible finite-dimensional linear representation of a connected locally compact group.

Lemma 7. Let $G$ be a locally compact group, $R$ the radical of $G$, and $S=G / R$ the semisimple quotient group of the group $G$ by its radical. The restriction of any (not necessarily continuous) irreducible finite-dimensional linear representation of $G$ to $R$ is a multiple of some central character of the group $R$, that is, a character $\lambda$ of $R$ such that $\lambda(r)=\lambda\left(g^{-1} r g\right)$ for all $g \in G, r \in R$; this character is automatically the identity on $[G, R]$ and can therefore be regarded as a character of the quotient group $R /[G, R]$.

Proof. Consider the restriction of a representation $\pi$ to the radical $R$. By part 2) of Corollary 5 , there is a basis in the space $E$ of the representation $\pi$ in which the restriction of the representation $\pi$ to $R$ is written as upper triangular matrices. Consequently there exists a common eigenvector $\xi \in E, \xi \neq 0$, for all operators $\pi(r), r \in R$, so that $\pi(r) \xi=\lambda(r) \xi$ for any $r \in R_{H}$, where $\lambda(r) \in \mathbb{C}^{*}=C \backslash\{0\}$ for any $r \in R_{H}$. Thus there exists a one-dimensional subspace $L \subset E$ that is invariant with respect to the restriction $\rho$ of the representation $\pi$ to $R$.

Consider a compact normal divisor $N$ in $G / R$ such that $L=(G / R) / N$, which is a (connected and automatically semisimple) Lie group. Let $N_{0}$ be the component of the identity in $N$. Then the quotient group $(G / R) / N_{0}$ contains the compact totally disconnected normal divisor $N / N_{0}$. Since $N / N_{0}$ is totally disconnected, it is central in $(G / R) / N_{0}$, where the locally compact group $G / R$ is semisimple. By Theorem $10.29(\mathrm{f})$ in 34 there exists a compact connected Abelian subgroup $M$ of $(G / R) / N_{0}$ containing $N / N_{0}$. By Corollary 1 in 9 ] there exists an infinitely divisible neighbourhood $W$ of the identity element in $M$. Let $O$ be the inverse image of the neighbourhood $W$ in $G / R$. Let $p \in \mathbb{N}$. Since the compact group $N_{0}$ is connected, it follows that $N_{0}$ is infinitely divisible, 
so that the set of $p$ th powers of elements of $O$ contains $N_{0}$. Furthermore we see that the set of $p$ th powers of elements of $O$ contains the set whose image in the quotient group $(G / R) / N_{0}$ is a neighbourhood of the identity element. Thus, the set of $p$ th powers of elements of $O$ is a generating set for $G / R$. By Lemma 6 this implies that all the operators of the representation $\pi$ are multiples of the identity operator in $E$,

$$
\pi(r)=\lambda(r) 1_{E} \text { for any } r \in R .
$$

In particular, the character $\lambda$ is central, that is, $\lambda(r)=\lambda\left(g^{-1} r g\right)$ for any $g \in G$ and $r \in R$. Hence it follows immediately that the function $\lambda$ is identically equal to one on $[G, R]$, where $R \supset[G, R] \supset R_{*}=[R, R]$, and therefore determines a character of the quotient group $R /[G, R]$. This completes the proof of Lemma 7

The proof of the next result is completely analogous to the proof of Lemma 7 since, according to Lemma 7, for any finite-dimensional representation of a connected locally compact group there exists a basis in the space of the representation in which the image of the radical is contained in the group of upper triangular matrices, and it remains to use the local divisibility of the group $G$ so as to verify that any element of a sufficiently small neighbourhood of the identity element in $G$ does not change the diagonal characters of the corresponding representation of the radical by upper triangular matrices. This result is well known for connected Lie groups; see [42, p. 194 and [38, Theorem XI.3.2, p. 128.

Lemma 8. Let $G$ be a connected locally compact group with radical $R$. For any (not necessarily continuous) finite-dimensional linear representation $\pi$ of $G$ there exists a basis in the space of the representation $\pi$ in which the family $\pi([G, R])$ is contained in the set of upper triangular matrices with ones on the principal diagonal.

It is clear that Lemmas 7 and 8 admit extensions to the case of general connected soluble normal divisors in a connected locally compact group.

We now recall one of the main results of the paper [9].

Theorem IV (see [9]). Let $G$ be a connected Lie group, let $G_{*}$ be the derived subgroup of $G$ and let $\pi$ be a locally bounded finite-dimensional representation of $G$. The restriction $\left.\pi\right|_{G_{*}}$ of the representation $\pi$ to $G_{*}$ is continuous on $G_{*}$ in the topology induced by the original topology of $G$.

This result on the automatic continuity of locally bounded finite-dimensional representations of connected locally compact groups is best possible, because any imperfect connected Lie group has a discontinuous locally bounded finite-dimensional representation. Here is the corresponding result.

Theorem V (see [9]). Let $G$ be a connected Lie group. The following conditions are equivalent.

(i) Any locally bounded finite-dimensional representation of $G$ is continuous.

(ii) Any locally bounded one-dimensional representation of $G$ is continuous.

(iii) Any unitary character of $G$ is continuous.

(iv) The group $G$ has no nontrivial locally bounded one-dimensional representations.

(v) The group $G$ has no nontrivial unitary characters.

(vi) The group $G$ has no nontrivial commutative quotient groups.

(vii) The group $G$ is perfect, that is, $G$ coincides with its derived subgroup $G_{*}$.

(viii) The Lie algebra $\mathfrak{g}$ of $G$ is perfect, that is, $\mathfrak{g}=[\mathfrak{g}, \mathfrak{g}]$.

(ix) The radical $\mathfrak{r}$ and the Levi subalgebra $\mathfrak{s}$ of the Lie algebra $\mathfrak{g}$ of $G$ (so that $\mathfrak{g}=\mathfrak{r} \rtimes \mathfrak{s}$ ) satisfy the condition $\mathfrak{r}=[\mathfrak{g}, \mathfrak{r}]$.

We shall also need the following result. 
Theorem VI (see [9]). Let $G$ be a topological group, and $\pi$ a finite-dimensional linear representation of $G$. If all the irreducible subquotient representations of $\pi$ are continuous (that is, the semisimple part of $\pi$ is continuous), then the representation $\pi$ itself is continuous.

The results listed above on automatic continuity relate, in the main, to Lie groups rather than the more general locally compact groups and, what is more, the property of automatic continuity of locally bounded finite-dimensional representations can break down (even in the narrower class of finite-dimensional unitary representations of a semisimple compact topological group) if that group is not a Lie group. In fact, if $G$ is the direct product of countably many copies of some semisimple Lie group $K, G=K^{\mathbb{N}}$ (say $K=\mathrm{SU}(2))$, then since $K$ is linear, that is, it has a faithful tautological finite-dimensional unitary representation, any matrix element $f_{i j}$ of any element of the group $f=\left\{f_{i j}\right\} \in G$ determines a (bounded) function $f_{i j}: \mathbb{N} \rightarrow \mathbb{C}$. Applying to each such function some fixed character $\chi$ of the Banach algebra $\mathrm{B}(\mathbb{N})$ of bounded complex-valued functions not defined by the map which takes the value of the function at some point of the set $\mathbb{N}$, we obtain the finite-dimensional unitary matrix representation $f=\left\{f_{i j}\right\} \rightarrow\left\{\chi\left(f_{i j}\right)\right\}$ of $G$, and this representation is clearly discontinuous.

Nevertheless, some assertions for general connected locally compact groups turn out to be consequences of theorems on the automatic continuity of Lie groups. We recall the description of the intersection of the kernels of continuous finite-dimensional unitary representations (the von Neumann kernel) and the intersection of the kernels of all (not necessarily continuous) finite-dimensional unitary representations ("the smallest von Neumann kernel") of a locally compact group [24]. Let $H=H(G)$ be the analytic subgroup of a connected locally compact group $G$ generated by all the noncompact simple analytic subgroups of $G$.

Theorem 9. The von Neumann kernel $\mathrm{vNk}(G)$ of an arbitrary connected locally compact group $G$ with radical $R$ is the closed normal divisor generated by $H$ and the normal divisor $[G, R]$ in $G$ generated by commutators of the form $\mathrm{grg}^{-1} r^{-1}$, where $g \in G$ and $r \in R$.

Theorem 10. The smallest von Neumann kernel $\operatorname{svNk}(G)$ of an arbitrary connected locally compact group $G$ with radical $R$ is the normal divisor generated by $H$ and the normal divisor $[G, R]$ in $G$ generated by commutators of the form grg $^{-1} r^{-1}$, where $g \in G$ and $r \in R$.

An example of a connected reductive Lie group $G$ with $\operatorname{vNk}(G) \neq \operatorname{svNk}(G)$ is also given in 24].

Before embarking on the study of kernels of universal representations of connected locally compact groups we recall certain characterizations of linear Lie groups (see [35. [43]).

Lemma 11. Let $G$ be a connected Lie group with Levi decomposition $G=S \cdot R$. Then the following conditions are equivalent:

(i) The group $G$ has a faithful continuous finite-dimensional linear representation.

(ii) The closure $\overline{R_{*}}$ of the group $R_{*}$ is simply connected (this condition holds if and only if the subgroup $R$ is closed and simply connected) and $S$ has a faithful continuous linear representation.

We also recall that a soluble connected Lie group $G$ has a faithful continuous finitedimensional linear representation if and only if the closure of the derived subgroup $G_{*}$ of $G$ is simply connected. This is a version of Theorem XVIII.3.2 in [38. According to Mal'tsev's classical result [4], the condition that a connected Lie group $G$ has a faithful continuous finite-dimensional linear representation is equivalent to the condition that 
both $R$ and the Levi subgroup $\Sigma$ of $G$ have faithful continuous finite-dimensional linear representations.

In this paper, which is in part a review article, we consider similar problems in the class of locally bounded linear representations of locally compact groups and, in particular, in the subclass of connected Lie goups, which will be used in the next section.

\section{LOCALLY BOUNDED LINEAR REPRESENTATION OF CONNECTED LIE GROUPS}

We begin the study of when connected locally compact groups have a linear representation with the special case relating to connected Lie groups. We recall that over 70 years ago, Birkhoff 35] proved that the Lie quotient group of the Heisenberg group by the normal divisor of the integer points in the centre has no (continuous or discontinuous) faithful finite-dimensional linear representations. Since then many works have appeared which are devoted to the study of Lie groups admitting faithful continuous finite-dimensional representations (see, for example, 36- 43]), whereas discontinuous representations have hardly been studied; Moskowitz thought that the investigation of discontinuous representations was hard 2 The following theorem answers the question of the existence of a faithful locally bounded finite-dimensional linear representation (this condition is different from the condition of representability in the class of locally bounded finite-dimensional linear representations) in the simplest possible way.

Theorem 12. A connected Lie group admits a faithful locally bounded (not necessarily continuous) finite-dimensional representation if and only if it admits a continuous faithful finite-dimensional representation, that is, if and only if it is a linear Lie group.

In what follows the symbol $Z(G)$ denotes the centre of the group $G, R=\operatorname{rad} G$ is the radical of a locally compact group $G$ (the largest soluble normal subgroup of $G$ ), $S$ denotes the semisimple quotient group by the radical, $S=G / R, \Sigma$ is the Levi subgroup of a connected Lie group, $G_{*}=[G, G]$ is the derived subgroup of $G$, and $R_{*}$ the radical of $G_{*}$.

Proof. We suppose that the connected Lie group $G$ has a faithful locally bounded linear representation $\pi$ in a complex vector space. Applying Theorem $\mathrm{V}$, we see that the restriction of $\pi$ to $\Sigma$ is automatically continuous and therefore $\Sigma$ is a linear group. By Lemma 11$]$ it remains to prove the assertion of the theorem under the additional assumption that $G$ is soluble. By part 2) of Corollary 5 we can suppose that the matrices of the representation $\pi$ are upper triangular, and therefore the matrix of the restriction of $\pi$ to $G_{*}$ is upper triangular with ones on the principal diagonal. Therefore the restriction of $\pi$ to $G_{*}$ is continuous according to Theorem VI and the image $\pi\left(G_{*}\right)$ of $G_{*}$ is an analytic subgroup of the simply connected nilpotent group of unipotent upper triangular matrices. Therefore $\pi\left(G_{*}\right)$ is closed and simply connected. Consequently $G_{*}$ is also simply connected. If the closure of $G_{*}$ contains a nontrivial compact subgroup, then the intersection of the closures of the complements of the bounded closed neighbourhoods of the identity element of $G_{*}$ contains elements of this compact subgroup, and then this intersection also contains the identity element of this compact subgroup, which is also an element of $G_{*}$. This property must be inherited by the continuous image $\pi\left(G_{*}\right)$; however, $\pi\left(G_{*}\right)$ is a subgroup such that the intersection of the closures of the complements of open neighbourhoods of the identity element cannot contain the identity element (because the norms of nilpotent matrices cannot be close to 1 at infinity). It follows that both parts of condition (ii) in Lemma 11 hold, so that $G$ is linear.

\footnotetext{
2 "Of course, in this form one cannot get necessary and sufficient conditions" [4].
} 


\section{Characterization of the Hochschild Kernel}

We recall that Hochschild 25] described the intersection of the kernels of all continuous finite-dimensional representations of a connected Lie group.

Definition 13. Let $G$ be a topological group. The Hochschild kernel of $G$ or the kernel of the universal representation $\operatorname{urk}(G)$ of $G$ is defined as the intersection of the kernels of all continuous finite-dimensional representations of $G$.

The following object is also of interest.

Definition 14. Let $G$ be a topological group. The kernel of locally bounded representations $\operatorname{lbrk}(G)$ of $G$ is defined as the intersection of the kernels of all (not necessarily continuous) locally bounded finite-dimensional representations of $G$.

It is obvious that $\operatorname{lbrk}(G)$ is a (not necessarily closed) normal divisor of $G$ contained in the closed normal divisor $\operatorname{urk}(G)$ of $G$. We summarize some results of the papers [36] -43, which clarifies the statement of the problem. According to Theorem 7.1 in [36], if $G$ is a Lie group, then the quotient group $G / \operatorname{urk}(G)$ is linear; that is, it has a faithful continuous finite-dimensional representation. Thus, $\operatorname{urk}(G)$ is the smallest closed normal divisor $N$ in the Lie group $G$ for which $G / N$ is isomorphic to a real-analytic subgroup of some full linear group.

Note that the group, which was studied by Birkhoff in [35, obviously satisfies the condition $\operatorname{urk}(G)=\operatorname{lbrk}(G)$, which suggests that this could turn out to be true even in the general case. But this is not the case. Here is an example (see [24]).

Example 15. We use the well-known example of a connected reductive Lie group with a nonclosed Levi subgroup (see, for example, [45], p. 256) and with derived subgroup that is dense in the group. Let $G_{0}$ be the universal covering group of the Lie group $\mathrm{SL}(2, \mathbb{R})$, and let $Z$ be the centre of $G_{0}$, which is isomorphic to the additive group of integers $\mathbb{Z}$. Let $\alpha \in \mathbb{R} \backslash \mathbb{Q}$, where $\mathbb{Q}$ is the subfield of rational numbers in $\mathbb{R}$. Let $\mathbb{T}=\mathbb{R} / \mathbb{Z}$, which is a one-dimensional torus. There exists a discrete central normal divisor $D$ in $\mathbb{R} \times Z$ (and simultaneously in $\mathbb{R} \times G_{0}$ ) such that the quotient group $\mathbb{R} \times Z / D$ is isomorphic to $\mathbb{T}$ and the image of a subgroup of $\{0\} \times Z$ that is dense in $\mathbb{T}$ (for example, we can take the kernel of the homomorphism $(t, \nu) \mapsto \exp 2 \pi i(t+\alpha \bar{\nu})$ for $D$, where $t \in \mathbb{R}, \nu \in Z$ are products of $\mathbb{R} \times Z$ by $\mathbb{T}$; here $\alpha$ is irrational and the map $\nu \mapsto \bar{\nu}$ is some isomorphism of $Z$ onto $\mathbb{Z}$ ( $\bar{\nu}$ is the integer corresponding to $\nu \in Z$ under this isomorphism). We can take the quotient group $G=\mathbb{R} \times G_{0} / D$ for the group $G$; let $C$ be the centre of $G$ and let $\rho$ be the canonical homomorphism of $\mathbb{R} \times G_{0}$ onto $G$. Clearly, $\rho(\mathbb{R} \times Z)=C, \rho\left(\{0\} \times G_{0}\right)=[G, G]$ and $\operatorname{vNk}(G)=G(G$ is minimal almost periodic $)$, whereas $\operatorname{svNk}(G))=[G, G]$.

As we see, the closure of $[G, G]$ is the entire group $G$. However, a connected Lie group, having a faithful finite-dimensional representation, has a closed Levi subgroup and a closed derived subgroup (see, for example, 45, Ch. 3, Exercise 41) while the quotient group $G$ can only satisfy these conditions if the corresponding normal divisor contains the subgroup $W=\rho\left(\mathbb{R} \times\left\{e_{Z}\right\}\right)$, so that $\operatorname{urk}(G)=W$. Here the group $[G, G]=\rho\left(\{0\} \times G_{0}\right)$ is obviously isomorphic to $G_{0}$, so that $\operatorname{lbrk}(G)=\operatorname{urk}\left(G_{0}\right) \subsetneq W$, because the quotient group $G / G_{0}$ (taken in the group-theoretic sense) is commutative and automatically has sufficiently many (possibly discontinuous) characters. As regards the group $\operatorname{urk}\left(G_{0}\right)$, it is equal to $2 Z$, as is well known.

Note that in this example the group $\operatorname{lbrk}(G)$ is not closed. Furthermore, $\operatorname{lbrk}(G) \neq\{e\}$ and $\operatorname{urk}(G) \neq\{e\}$. This is a general fact: if the $\operatorname{groups} \operatorname{lbrk}(G)$ and $\operatorname{urk}(G)$ are not the same, then they are both nontrivial; see Theorem 12 above. 
We now prove a theorem on continuous representability for a connected locally compact group. This theorem asserts that a connected locally compact group $G$ has sufficiently many continuous linear representations in finite-dimensional vector spaces, or equivalently the condition $\operatorname{urk}(G)=\left\{e_{G}\right\}$ is satisfied, if and only if $G$ is a projective limit of connected linear Lie groups. We also show that these conditions are equivalent to a combination of two conditions, namely, that the largest noncompact (analytic) semisimple Lie subgroup $S_{1}$ of $G$ is a linear Lie group (so that the corresponding group $\operatorname{urk}\left(S_{1}\right)$ is trivial) and the (unique) maximal compact subgroup $B$ of the closure in $G$ of the radical $\operatorname{rad}\left(G^{\prime}\right)$ of the derived subgroup $G^{\prime}=[G, G]$ of $G$ is also trivial. We now state this result on the equivalence of certain properties of a connected locally compact group.

Theorem 1. Let $G$ be a connected locally compact group. The following conditions are equivalent:

(i) $G$ has sufficiently many continuous linear representations in finite-dimensional vector spaces, that is, the family of finite-dimensional linear representations of $G$ separates the points of $G$;

(ii) $\operatorname{urk}(G)=\left\{e_{G}\right\}$;

(iii) $G$ is a projective limit of connected linear Lie groups;

(iv) the largest noncompact (analytic) semisimple Lie subgroup $S_{1}$ in $G$ is a linear Lie group (this means, of course, that the corresponding group $\operatorname{urk}\left(S_{1}\right)$ is trivial) and the (unique) maximal compact subgroup $B$ of the closure in $G$ of the radical $\operatorname{rad}\left(G^{\prime}\right)$ of the derived subgroup $G^{\prime}$ of $G$ is also trivial.

Proof. It is obvious that (iii) $\Rightarrow$ (i) $\Leftrightarrow$ (ii). We shall prove the chain of implications (i) $\Rightarrow$ (iv) $\Rightarrow$ (iii).

If condition (i) holds, then not only $G$ but also $S$ has sufficiently many continuous linear representations in finite-dimensional vector spaces. According to the equivalence (i) $\Leftrightarrow$ (ii) (see above), it follows that $\operatorname{urk}\left(S_{1}\right)=\left\{e_{S_{1}}\right\}$. Consequently, the closure of $\operatorname{urk}\left(S_{1}\right)$ in $G$ is also the identity. Let $U$ be a given neighbourhood of the identity element in $G$, and $N$ a normal divisor in $G$ contained in $U$ such that $G / N$ is a connected Lie group. We apply Theorem II (Hochschild's main theorem on the kernel of the universal representation of connected Lie groups) to the quotient Lie group $H=G / N$. Since the groups $S_{1}(G)$ and $S_{1}(G / N)$ are naturally isomorphic, it follows that $H=G / N$ is a Lie group whose Levi subgroup $S$ is linear, so that the group $A=\operatorname{urk}(S)$ is trivial, $A=\operatorname{urk}(S)=\left\{e_{S}\right\}$. Then the closure $\mathcal{A}$ of $A$ is also trivial, $\mathcal{A}=\left\{e_{G}\right\}$. Again by Theorem II, the Lie group $H$ has a faithful continuous finite-dimensional representation in this case (or equivalently, using Theorem 7.1 in [36] on connected Lie groups, it has sufficiently many continuous linear representations in finite-dimensional vector spaces) if and only if the subgroup $P$ of $G$, which for $\operatorname{urk}(S)=\left\{e_{S}\right\}$ coincides with the (unique) maximal compact subgroup $B$ of the closure in $G$ of the radical $\operatorname{rad}\left(G^{\prime}\right)$ of the derived subgroup $G^{\prime}$ of $G$, is trivial.

However, if the maximal compact subgroup $B$ of the closure in $G$ of $\operatorname{rad}\left(G^{\prime}\right)$ is nontrivial, then there exists a neighbourhood $V$ of the identity element in $G$ that does not contain elements of $B$. In this case the image of $B$ in $G / N$, where $N$ is a compact normal divisor in $G$ contained in $V$, is a nontrivial compact subgroup of the closure in $H$ of the image of $\operatorname{rad}\left(G^{\prime}\right)$ in $H$. On the other hand, since the normal divisor $N$ is compact, it follows from Corollary 3.8 in 7 in combination with $\S 3$ in 14 that the image in $H$ of $\operatorname{rad}\left(G^{\prime}\right)$ coincides with $\operatorname{rad}\left(H^{\prime}\right)$ since the radicals in $G$ and $H$ are automatically closed. Thus, if $B$ is nontrivial, then the Hochschild kernel $\operatorname{urk}(H)$ of any quotient Lie group of the form $H=G / N$ is nontrivial since it contains the nontrivial image of $B$ in $H$. Thus $B \subset \operatorname{urk}(G)$ and the family of continuous finite-dimensional linear representations 
of $G$ does not separate points of the group. This completes the proof of the implication (i) $\Rightarrow$ (iv).

To complete the proof of the theorem it suffices for us to prove the implication (iv) $\Rightarrow$ (iii). Suppose that the groups $\mathcal{A}=\mathcal{A}(g)$ and $B=B(G)$ are trivial. Consider a neighbourhood $U$ of the element $e_{G}$ in $G$. We will find a normal divisor $N \subset U$ such that $H=G / N$ is a Lie group. As we have already seen, in this case we also have $\mathcal{A}(H)=\left\{e_{H}\right\}$. We suppose that for this $N$ there exists a nontrivial compact group $B(H)$ of the closure in $H$ of the subgroup $\operatorname{rad}\left(H^{\prime}\right)$. Since $B$ is part of the kernel of the universal representation and the kernel of the adjoint representation coincides with the centre of the group, it follows that $B$ is central and therefore is a normal divisor in $G$. Consequently (recall that $N$ is compact), the inverse image $\widetilde{B(H)}$ of $B(H)$ in $G$ is a compact normal divisor in $G$ and this compact group $\widetilde{B(H)}$ is commutative modulo $N$; that is, the derived subgroup of this group is contained in $N$.

The intersection $C$ of all compact normal divisors of the form $\widetilde{B(G / N)}$ in $G$ (with respect to all compact normal divisors $N$ such that the quotient group $G / N$ is a Lie group) is a compact subgroup of $G$. The subgroup $C$ is commutative, since the derived subgroup of $C$ is contained in the intersection of all groups of the form $N$ and this intersection is the identity. Furthermore, $B(H)$ is a compact subgroup of the closure in $H$ of $\operatorname{rad}\left(H^{\prime}\right)$. Since, as with the radical of the group, the image of $\operatorname{rad}\left(G^{\prime}\right)$ is $\operatorname{rad}\left(H^{\prime}\right)$ and the inverse image of $\operatorname{rad}\left(H^{\prime}\right)$ is $\operatorname{rad}\left(G^{\prime}\right) N$, we conclude that the compact subgroup $\widehat{B(G / N)}$ (which lies in $\operatorname{rad}\left(H^{\prime}\right)$ ) is contained in the product of the closure $\overline{\operatorname{rad}\left(G^{\prime}\right)}$ of $\left.\operatorname{rad}\left(G^{\prime}\right)\right)$ and the normal divisor $N$. Thus, the intersection $C$ of all compact normal divisors of the form $\widehat{B(G / N)}$ in $G$ is contained in the intersection of the products $\overline{\operatorname{rad}\left(G^{\prime}\right)} N$, that is, in the closure in $G$ of $\operatorname{rad}\left(G^{\prime}\right)$, the radical of the derived subgroup $G^{\prime}=[G, G]$ of $G$.

Furthermore, the quotient group $G / \widetilde{B(G / N)}$ is naturally isomorphic to the quotient

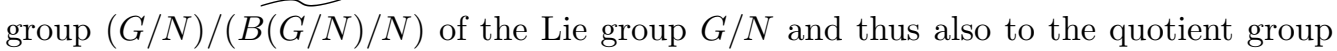
$(G / N) / B(G / N)$ and, by construction and by Hochschild's Theorem II above, the quotient Lie group $(G / N) / B(G / N)$ (and with it the quotient Lie group $G / \widetilde{B(G / N)}$ ) is a linear Lie group. Consider the common kernel $C=\bigcap_{N} \widetilde{B(G / N)}$ (introduced above) of all linear representations of $G$ in finite-dimensional vector spaces obtained in this way. Since all the groups $\widetilde{B(G / N)}$ are compact normal divisors, it follows that $C$ too is a compact normal divisor in $G$. We saw above that it is also commutative and that $C$ is contained in the closure in $G$ of $\operatorname{rad}\left(G^{\prime}\right)$. In view of condition (iv) the group $C$ is the identity. Since, by the construction of the group $C$, any element of $G$ not belonging to $C$ goes over to a nonidentity element in some continuous finite-dimensional representation $\pi$ of $G$ and since all quotient Lie groups of the form $G / \widetilde{B(G / N)}$ are linear, the group $G=G / C$ turns out to be the projective limit of linear Lie groups of the form $G / \widehat{B(G / N)}$. This completes the proof of Theorem 1 .

We now prove the result on the Hochschild kernel urk $(G)$ of a connected locally compact group $G$, which we stated in the introduction.

Theorem 2. Let $G$ be a connected locally compact group, $U$ a given neighbourhood of the identity in $G, N \subset U$ a compact (not necessarily connected) normal divisor such that the quotient group $L=G / N$ is a connected Lie group and $G$ is the image of some homomorphism $\psi: \widetilde{L} \times N \rightarrow G$ with discrete kernel, where $\widetilde{L}$ is the universal covering of the Lie group L. Let $S_{\widetilde{L}}$ be the Levi subgroup of $\widetilde{L}$ and let $A$ be the closure (in $G$ ) 
of $\operatorname{urk}(S)$ (see Theorem I). Then $\operatorname{urk}(G)$ coincides with the closed (normal) subgroup $P$ of $G$ containing $A$ for which the quotient group $P / A$ is the (unique) maximal compact subgroup of the closure (in $G / A)$ of the radical of the derived subgroup $(G / A)_{*}$ of $G / A$.

Proof. Let $S_{\widetilde{L}}$ be the Levi subgroup of $\widetilde{L}$ and let $A$ be the closure in $G$ of the subgroup $\operatorname{urk}\left(\psi\left(S_{\widetilde{L}}\right)\right)$ described in Theorem I. Since any continuous finite-dimensional representation of $G$ is the identity on the subgroup $\operatorname{urk}\left(\psi\left(S_{\widetilde{L}}\right)\right)$, it follows that the adjoint representation of any quotient Lie group $G / N$ is also the identity on the subgroup $\operatorname{urk}\left(\psi\left(S_{\widetilde{L}}\right)\right.$ ). Thus, the image of any element of the subgroup $\operatorname{urk}\left(\psi\left(S_{\widetilde{L}}\right)\right.$ lies in the centre of $G / N$ and consequently the element itself lies in the centre of $G$. Therefore the subgroup $A$ is also central; in particular, it is a closed normal divisor in $G$. Since all continuous finite-dimensional linear representations of $G$ are trivial on $A$, we can go over to the quotient group $G / A$ and regard continuous finite-dimensional linear representations of $G$ as continuous finite-dimensional linear representations of $G / A$. We shall simplify the notation by denoting the group $G / A$ by the letter $G$; then it suffices to observe that $\operatorname{urk}\left(\psi\left(S_{L / \widetilde{(L \cap A)}}\right)\right)=\{e\}$ by construction, and then the closure of this identity group is the identity. It remains to prove that in the case under consideration we have the equality $\operatorname{urk}(G)=C$, where $C$ is the (unique) maximal compact subgroup of the closure in $G$ of the radical of the derived subgroup $G_{*}$ of $G$. (Recall that after factorization by $A$, a maximal semisimple analytic subgroup of $G$ without compact factors becomes a linear Lie group.)

We now remark that the closed subgroup $\overline{[G, R]}$ of $G$ is a connected locally compact group. Consequently, the maximal compact subgroup $C$ of $\overline{[G, R]}$ exists and is a connected group (see [26, Theorem 13). Furthermore, since $C \subset \overline{[G, R]} \subset R$, we see that $C$ is simultaneously compact, connected and soluble. Therefore $C$ is a compact Abelian group. Since the restriction to $C$ of any continuous finite-dimensional linear representation of $G$ is a direct sum of one-dimensional (and therefore irreducible) representations, it follows that any continuous finite-dimensional linear representation of $G$ is the identity on the subgroup $C$ by Lemma 8, Repeating the corresponding argument in the previous paragraph we see that the subgroup $C$ is central; in particular, it too is a normal divisor in $G$. Since all maximal compact subgroups $C$ in any connected locally compact group are conjugate, in view of the centrality they all coincide. Thus the group $C$ is uniquely defined and is the unique maximal compact group of $\overline{[G, R]}$.

Thus we have proved that $\operatorname{urk}(G)$ contains a closed normal divisor $P$ in $G$ whose quotient group by $A$ is the unique maximal compact subgroup of $\overline{[G, R]}$.

We now prove the converse assertion. We consider the quotient group of $G$ by the closed normal divisor $P$ of $G$ generated by the subgroup $A$ and the unique maximal compact subgroup of $\overline{[G, R]}$. We again denote this group by $G$; then $A=\{e\}$ and the maximal compact subgroup of the closure $\overline{[G, R]}$ is the identity. (In fact, the group $C(G / C)$ for the quotient group by $C$ is compact and Abelian, so that the extension of this group by $C$ is compact, connected and soluble and is contained in the closure $\overline{[G, R]}$ and is therefore Abelian, and $C$ is maximal among the compact subgroups of the closure $\overline{[G, R]}$.) It then follows from Theorem 1 that the quotient group $G / P$ has sufficiently many continuous linear finite-dimensional representations. This completes the proof of Theorem 2 ,

We shall not give here a description of the intersection of the kernels of all locally bounded representations for any arbitrary connected locally compact group. However in $\S 5$, which follows, we obtain a certain generalization of the Hochschild characterization in the form of sufficient conditions for the equality $\operatorname{urk}(G)=\operatorname{lbrk}(G)$ to hold, while in 
$\S 6$ we give a complete description of the intersection of the kernels of all locally bounded representations for an arbitrary connected reductive Lie group.

\section{A version of Hochschild's Theorem}

It is clear that $\operatorname{lbrk}(G) \subset \operatorname{urk}(G)$. Of course, $\operatorname{urk}(G)$ is a closed normal subgroup of $G$ 48. and, according to Theorem 7.1 in 36, the quotient group $G / \operatorname{urk}(G)$ has a faithful continuous finite-dimensional representation. Thus, $\operatorname{urk}(G)$ is the smallest closed normal divisor $P$ in $G$ satisfying the condition that $G / P$ is isomorphic to a real-analytic subgroup of a full linear group.

The example above, which gave a connected reductive Lie group with $\operatorname{urk}(G) \subsetneq$ $\operatorname{lbrk}(G)$, means that in any theorem dealing with sufficient conditions for the groups $\operatorname{lbrk}(G)$ and $\operatorname{urk}(G)$ to coincide, some extra conditions are unavoidable. Instead of the continuous finite-dimensional representations that feature in Hochschild's theorem we consider finite-dimensional representations that are locally bounded and continuous on the closure of the kernel of the universal representation of some Levi subgroup of the entire group, and on the closure of the radical of the derived subgroup. Thus, this theorem generalizes Hochschild's characterization.

Theorem 16. Let $G$ be a connected Lie group, and $S$ a maximal semisimple analytic subgroup of $G$. Let $A$ be the closure in $G$ of the group $\operatorname{urk}(S)$. Then the intersection $L_{G}$ of the kernels of all locally bounded finite-dimensional representations of $G$ (in complex vector spaces) that are continuous on $A$ and on the closure of $\operatorname{rad}\left(G_{*}\right)$ in $G$ is a normal divisor $P$ in $G$ containing $A$ for which the quotient group $P / A$ is the (unique) maximal compact subgroup of the closure in $G / A$ of the radical of the derived subgroup $(G / A)_{*}$ of $G / A$. If $G$ is perfect (that is, the derived subgroup of $G$ is equal to $G$ ) and also if (1) the Levi subgroup $S$ is closed in $G$ or the kernel of the universal representation of $S$ is closed in $G$ and (2) the closure of the radical $\operatorname{rad}\left(G_{*}\right)$ in $G$ is contained in the derived subgroup $G_{*}$ of $G$, then $L_{G}=\operatorname{vNk}(G)$, and consequently in these cases the group $M_{G}$ coincides with $\operatorname{vNk}(G)$.

Proof. Let $R=\operatorname{rad}(G)$ be the radical of the connected Lie group $G$, and $\pi$ a (generally speaking discontinuous) finite-dimensional representation of $G$. By Theorem 2 the restriction of $\pi$ to $S$ is automatically continuous; therefore the representation $\pi$ maps the normal divisor $\operatorname{vNk}(S)$ of $S$ into the identity operator in the space of the representation $\pi$. By assumption, the representation $\pi$ is continuous on the closure $A$ of the group $\operatorname{vNk}(S)$ in $G$ and therefore the representation $\pi$ is trivial not only on $\operatorname{vNk}(S)$ but also on the whole of $A$. We point out that any Lie group has an adjoint representation whose kernel coincides with the centre $Z_{G}$ of the group $G$. Consequently vNk $(S) \subset Z_{G}$, where $Z_{G}$ is automatically closed. Thus, the closure $A$ of $\mathrm{vNk}(S)$ in $G$ is also contained in $Z_{G}$ and therefore the group $A$ is central. Thus, $A$ is a closed normal divisor in $G$.

It follows that any finite-dimensional representation of $G$ in a complex vector space satisfying the conditions of Theorem [16] is trivial on $A$. By going over to the group $G / A$ we can regard any finite-dimensional representation of $G$ satisfying the conditions of Theorem 16 (and not necessarily continuous) as a representation of the group $G / A$. For this reason we can suppose that the given group $G$ has a linear Levi subgroup $S$ and is trivial on $A$.

It remains to prove that any finite-dimensional linear representation $\pi$ of such a group $G$ in a complex vector space satisfying the conditions of Theorem 16 is the identity on any compact subgroup $Q$ of the closure in $G$ of $\operatorname{rad} G_{*}$, the radical of the derived subgroup $G_{*}$ of $G$. For brevity we denote $\overline{\operatorname{rad} G_{*}}$ by the symbol $O$. Since the restriction of $\pi$ to $G_{*}$ is continuous by Theorem IV and the identity on each irreducible subrepresentation of $\pi$ 
by Lemma 17, it follows that the restriction of $\pi$ to $\operatorname{rad} G_{*}$ is continuous and is represented by upper triangular matrices with ones on the principal diagonal (see [9, [49]), and it follows from our condition of continuity that the restriction of $\pi$ to $O$ is also continuous and represented by upper triangular matrices with ones on the principal diagonal. Thus, the representation $\pi$ is trivial on each compact subgroup of $O$, which proves that $L_{G}$ contains a maximal compact subgroup of $O$. Consequently, $L_{G} \supset \mathrm{vNk}(G)$. Since clearly $L_{G} \subset \operatorname{vNk}(G)$, the proof is complete.

\section{A Version of Hochschild's TheOrEm on the KERNEL FOR LOCALLY BOUNDED REPRESENTATIONS OF CONNECTED REDUCTIVE LIE GROUPS}

In this section we give a description of the intersection of the kernels of all locally bounded representations $\operatorname{lbrk}(G)$ for an arbitrary connected reductive Lie group $G$.

Theorem 17. Let $G$ be a connected reductive Lie group, and $S$ a maximal semisimple analytic subgroup of $G$. Let $A$ be the "linearizer" of $S$, that is, the group $\operatorname{urk}(S)$, characterized in Theorem I. Then the group $\operatorname{lbrk}(G)$, which is the intersection of all (not necessarily continuous) locally bounded finite-dimensional representations of $G$ in complex vector spaces, coincides with A.

Proof. Let $R=\operatorname{rad} G$ be the Abelian radical of the connected reductive Lie group $G$. Suppose that $\pi$ is a (generally speaking discontinuous) locally bounded finite-dimensional representation of $G$. By van der Waerden's theorem [2] (see also 9]) the restriction of $\pi$ to $S$ is automatically continuous (hence, in particular, $\operatorname{urk}(S)=\operatorname{lbrk}(S)$ ) and therefore the representation $\pi$ maps the normal divisor $A$ of $S$ into the identity operator in the space of the representation $\pi$.

We recall that the kernel of the adjoint representation of any Lie group is the centre of the group. Consequently it follows from the obvious inclusion $A \subset \operatorname{urk}(G)$ that $A$ is a subgroup of the centre of $G$. Since any (continuous) finite-dimensional representation of the semisimple Lie group $S$ is completely reducible, it follows that any locally bounded representation of $S$ is a direct sum of irreducible representations.

Let $B=S \cap R$. Since $S$ does not have any connected soluble normal divisors, it follows that $B$ is a discrete normal divisor of $S$. Consequently $B$ is a subgroup of the centre $Z_{S}$ of $S$.

We return to our investigation of a locally bounded finite-dimensional representation of $G$ and restrict this representation to the subgroup $S$. As we remarked above, this restriction is a direct sum of irreducible representations of $S$. In particular, any element of the centre $Z_{S}$ of $S$ is taken to a scalar operator in the subspace of any irreducible subrepresentation.

This property means that any element of the set-theoretic difference $Z_{S} \backslash A$ and, in particular, any element of the set-theoretic difference $B \backslash A$ is taken to a nonidentity operator in the space of the representation for some locally bounded finite-dimensional linear representation of $G$.

We now consider the Abelian group $R$ and endow it with the discrete topology. We claim that any locally bounded finite-dimensional linear representation of $S$ can be extended to a locally bounded finite-dimensional linear representation of $G$. It suffices to establish this claim for irreducible representations. If the representation under consideration is irreducible, then the restriction of this representation to the intersection $B=S \cap R$ (discrete in $S$ and therefore central in $S$ ) is determined by some character of $B$ by Schur's lemma. According to Theorem 24.12 in $[50$ this character can be extended to a character of the entire (Abelian) group $R$. Since elements of the groups $S$ and $R$ commute (because $R$ is central by hypothesis), this extension of the character determines the desired extension of the representation of the entire group $S$. 
Hence it follows that any element of the complement $S \backslash A$ has a nontrivial image in some locally bounded finite-dimensional linear representation of $G$. Elements of the set $R \backslash B$ also have a nontrivial image in some locally bounded finite-dimensional linear representation of $G$, because the quotient group $R / B$ is automatically Abelian, so that it has sufficiently many one-dimensional (unitary) characters. This completes the proof of Theorem 17

We point out the following obvious corollary.

Corollary 18. A connected reductive Lie group $G$ with Levi subgroup $S$ satisfies the condition $\operatorname{urk}(G)=\operatorname{lbrk}(G)$ if and only if $\operatorname{urk}(S)$ is closed in $G$.

The proof of Theorem 17 throws up difficulties which relate to characterizations of the kernel $\operatorname{lbrk}(G)$ for a connected Lie group $G$ of general form. These are even more in evidence in the case of an arbitrary connected locally compact group. The simplifying role of the assumption that the group $G$ in question is reductive (and therefore the role of the assumption that the radical is central) is clearly visible. We shall return to the problem of characterizing the $\operatorname{kernel} \operatorname{lbrk}(G)$ for a connected Lie group $G$ in another publication.

\section{REFERENCES}

[1] É. Cartan, Sur les représentations linéaires des groupes clos, Comment. Math. Helv. 2 (1930), no. 1, 269-283. MR 1509418

[2] B.L. van der Waerden, Stetigkeitssätze für halbeinfache Liesche Gruppen, Math. Z. 36 (1933), 780-786. MR 1545369

[3] J. D. Jawson, Intrinsic topologies in topological lattices and semilattices, Pacific J. Math. 44 (1973), 593-602. MR0318031 (47:6580)

[4] W.W. Comfort, Topological groups, Handbook of set-theoretic topology, North-Holland, Amsterdam-New York, 1984, pp. 1143-1263. MR776643 (86g:22001)

[5] A. Pilay, An application of model theory to real and p-adic algebraic groups, J. Algebra 126 (1989), no. 1, 139-146. MR1023289(90m:03061)

[6] K.H. Hofmann and S.A. Morris, The structure of compact groups, de Gruyter, Berlin, 1998. MR $1646190(99 \mathrm{k}: 22001)$

[7] W.W. Comfort, D. Remus and H. Szambien, Extending ring topologies, J. Algebra 232 (2000), no. 1, 21-47. MR1783911 (2001i:16081)

[8] J.E. Hart and K. Kunen, Bohr compactifications of non-abelian groups, Topology Proc. 26 (2001/02), no. 2, 593-626. MR2032839(2005c:22012)

[9] A.I. Shtern, A version of van der Waerden's theorem and a proof of Mishchenko's conjecture for homomorphisms of locally compact groups, Izv. Ross. Akad. Nauk Ser. Mat. 72 (2008), no. 1, 183-224; English transl., Izv. Math. 72 (2008), no. 1, 169-205. MR2394977 (2009h:22004)

[10] D. Remus, Private communication (2010).

[11] W. W. Comfort and L. C. Robertson, Images and quotients of $\mathrm{SO}(3, \mathbf{R})$ : remarks on a theorem of van der Waerden, Rocky Mountain J. Math. 17 (1987), no. 1, 1-13. MR882879 (88f:22012)

[12] A. Borel, Essays in the history of Lie groups and algebraic groups, Amer. Math. Soc., Providence, RI: London Mathematical Society, Cambridge, 2001. MR.1847105 (2002g:01010)

[13] O. Schreier and B. L. van der Waerden, Die Automorphismen der projektiven Gruppen, Abh. Math. Sem. Hamburg 6 (1928), 303-322.

[14] H. Freudenthal, Die Topologie der Lieschen Gruppen als algebraisches Phänomenon I , Ann. of Math. (2) 42 (1941), 1051-1074. MR0005740 (3:198a)

[15] W. T. van Est, Dense imbeddings of Lie groups, Nederl. Akad. Wetensch. Proc. Ser. A. 54 = Indagationes Math. 13 (1951), 321-328. MR0044530(13:432c)

[16] W. T. van Est, Dense imbeddings of Lie groups II (I,II), Nederl. Akad. Wetensch. Proc. Ser. A. 55 = Indagationes Math. 14 (1952), 256-266; 267-274. MR0049202 (14:135b)

[17] M. Gotô, Dense imbeddings of topological groups, Proc. Amer. Math. Soc. 4 (1953), 653-655. MR0056613(15:101c)

[18] M. Gotô, Dense imbeddings of locally compact connected groups, Ann. of Math. (2) 61 (1955), 154-169. MR0065567 (16:447b) 
[19] J. Tits, Homomorphismes "abstraits" de groupes de Lie, Symposia Mathematica XIII (Convegnodi Gruppi e loro Rappresentazioni, INDAM, Rome, 1972), Academic Press, London (1974), 479-499. MR0379749(52:654)

[20] A. Borel and J. Tits, Homomorphismes "abstraits" de groupes algébriques simples, Ann. of Math. (2) 97 (1973), 499-571. MR0316587(47:5134)

[21] D. James, W. Waterhouse and B. Weisfeiler, Abstract homomorphisms of algebraic groups: problems and bibliography; Bibliography: Abstract homomorphisms of algebraic groups, Comm. Algebra 9 (1981), no. 1, 95-114. MR599074 (81m:20059)

[22] Yu. Chen, Homomorphisms from linear groups over division rings to algebraic groups, Group theory, Beijing (1984), Springer, Berlin, New York, 1986 (Lecture Notes in Math. 1185, 231-265). MR.842446 (87i:20080)

[23] G. M. Seitz, Abstract homomorphisms of algebraic groups, J. London Math. Soc. (2) 56 (1997), no. 1, 104-124. MR1462829 (99b:20077)

[24] A.I. Shtern, The structure of homomorphisms of connected locally compact groups and compact groups, Izv. Ross. Akad. Nauk Ser. Mat., to appear.

[25] G.P. Hochschild, The universal representation kernel of a Lie group, Proc. Amer. Math. Soc. 11 (1960), 625-629. MR0123640 (23:A965)

[26] K. Iwasawa, On some types of topological groups, Ann. of Math. (2) 50 (1949), 507-558. MR0029911 $(10: 679 a)$

[27] A. L. T. Paterson, Amenability, Math. Surveys and Monographs 29 (1988), Amer. Math. Soc., Providence, RI. MR.961261 (90e:43001)

[28] A.I. Shtern, Applications of automatic continuity results to analogs of the Freudenthal-Weil and Hochschild theorems, Adv. Stud. Contemp. Math. (Kyungshang), 20 (2010), no. 2, $203-212$. MR2656974(2011e:22014)

[29] A.I. Shtern, Hochschild kernel for locally bounded finite-dimensional representations of a connected reductive Lie group, Proc. Jangjeon Math. Soc. 13 (2010), no. 2, 127-132. MR2676681 (2011g:22013)

[30] A.I. Shtern, Von Neumann kernel of a connected locally compact group, revisited, Adv. Stud. Contemp. Math. (Kyungshang) 20 (2010), no. 3, 313-318. MR2676901 (2011e:22008)

[31] A. I. Shtern, Homomorphic images of connected Lie groups in compact groups, Adv. Stud. Contemp. Math. (Kyungshang) 20 (2010), no. 1, 1-6. MR2597987 (2011e:22013)

[32] A.I. Shtern, Connected Lie groups having faithful locally bounded (not necessarily continuous) finite-dimensional representations, Russ. J. Math. Phys. 16 (2009), no. 4, 566-567. MR2587813 (2011a:22007)

[33] A.I. Shtern, Freudenthal-Weil theorem for arbitrary enbeddings of connected Lie groups in compact groups, Adv. Stud. Contemp. Math. (Kyungshang) 19 (2009), no. 2, 157-164. MR2566913 (2011b:22008)

[34] K. H. Hofmann and S. A. Morris, The Lie theory of connected pro-Lie groups, European Mathematical Society, Berlin, 2007. MR2337107(2008h:22001)

[35] G. Birkhoff, Lie groups simply isomorphic with no linear group, Bull. Amer. Math. Soc. 42 (1936) 883-888. MR 1563459

[36] M. Gotô, Faithful representations of Lie groups, Math. Japonicae I (1948), 107-119; II 1 (1950), 91-107. MR0029919(10:681a) MR0038981(12:479d)

[37] Harish-Chandra, On faithful representations of Lie groups, Proc. Amer. Math. Soc. 1 (1950), 205210. MR.0034396(11:579h)

[38] G.P. Hochschild, The Structure of Lie Groups, Holden-Day, San Francisco-London-Amsterdam, 1965. MR0207883 (34:7696)

[39] G.P. Hochschild, Complexification of real analytic groups, Trans. Amer. Math. Soc. 125 (1966), 406-413. MR0206141 (34:5966)

[40] G. P. Hochschild and G. D. Mostow, Extensions of representations of Lie groups and Lie algebras, I. Amer. J. Math. 79 (1957), 924-942. MR0103941 (21:2703)

[41] D. Luminet and A. Valette, Faithful uniformly continuous representations of Lie groups, J. London Math. Soc. (2) 49 (1994), no. 1, 100-108. MR1253015 (95c:22028)

[42] M. Moskowitz, A remark on faithful representations, Atti Accad. Naz. Lincei Rend. Cl. Sci. Fis. Mat. Natur. (8) 52 (1972), 829-831. MR0327979 (48:6321)

[43] M. Moskowitz, Faithful representations and a local property of Lie groups, Math. Z. 143 (1975), 193-198. MR0374342 (51:10542)

[44] A. I. Mal'tsev, Linear connected locally closed groups, Dokl. Akad. Nauk SSSR 40 (1943), no. 3, 108-110; S. R. Dokl. Acad. Sci. URSS (N.S.) 40 (1943), 87-89. 
[45] V.S. Varadarajan, Lie groups, Lie algebras, and their representations, Prentice-Hall, Englewood Cliffs, NJ, 1974. MR0376938 (51:13113)

[46] N. W. Rickert, Some properties of locally compact groups, J. Austral. Math. Soc. 7 (1967), $433-454$. MR0219656(36:2735)

[47] A. Weil, L'intégration dans les groupes topologiques et ses applications, 2nd ed., Hermann, Paris, 1953.

[48] J. von Neumann, Almost periodic functions in a group, I, Trans. Amer. Math. Soc. 36 (1934), 445-492. MR 1501752

[49] A.I. Shtern, Finite-dimensional quasi-representations of connected Lie groups and Mishchenko's conjecture 13 (2007), no. 7, 85-225; English transl., J. Math. Sci. 159 (2009), no. 5, 653-751. MR2475577 (2010c:22020)

[50] E. Hewitt and K.A. Ross, Abstract harmonic analysis, Springer-Verlag, Berlin-New York, 1979. MR.551496(81k:43001)

Faculty of Mechanics and Mathematics, Moscow State University, Russia

E-mail address: ashtern@member.ams.org

Translated by G. G. GOULD 